\title{
A super Frobenius formula for the characters of Iwahori-Hecke algebras
}

\author{
Hideo Mitsuhashi* \\ Department of Mechanical Engineering \\ Tomakomai National College of Technology \\ 443 Nishikioka, Tomakomai, Hokkaido, 059-1275, Japan
}

\begin{abstract}
In this paper, we establish a super Frobenius formula for the characters of Iwahori-Hecke algebras. We define Hall-Littlewood supersymmetric functions in a standard manner to make supersymmetric functions from symmetric functions, and investigate some properties of supersymmetric functions. Based on Schur-Weyl reciprocity between Iwahori-Hecke algebras and the general quantum super algebras, which was obtained in [8, we derive that one of several types of Hall-Littlewood supersymmetric functions, up to constant, generates the values of the irreducible characters of Iwahori-Hecke algebras at the elements corresponding to cycle permutations. Our formula in this article includes both the ordinary quantum case $(n=0)$ that was obtained in [10] and the classical super case $(q \rightarrow 1)$.
\end{abstract}

\section{Introduction}

The Frobenius formula is one of powerful methods to compute the irreducible characters of symmetric groups. It is based on Schur-Weyl reciprocity; there are actions of symmetric groups and of general linear groups which generate the full centralizer of each other. Schur-Weyl reciprocity has been extended to various groups and algebras up to the present. Among them, two remarkable extensions for us are the super type extension $([2,[12])$ and the quantum type one ([4]). In our paper [8], we established Schur-Weyl reciprocity between the Iwahori-Hecke algebra $\mathscr{H}_{\mathbb{Q}(q), r}(q)$ and the quantum super algebra $U_{q}^{\sigma}(\mathfrak{g l}(m, n))$, which unifies Schur-Weyl reciprocity of super type and that of quantum type. In [10, Ram gave a (ordinary) Frobenius formula for the characters of the Iwahori-Hecke algebras of type $A$, which is based on the Schur-Weyl reciprocity between the Iwahori-Hecke algebra of type $A$ and the quantum enveloping algebra of $\mathfrak{g l}_{n}$ that was given in [4. An extension of Frobenius formula to Ariki-Koike algebras, that are Hecke algebras associated to complex reflection groups $G(r, 1, n)$, is found in [13. In this paper,

*E-mail : mitsu@gt.tomakomai-ct.ac.jp 
we give a super Frobenius formula for the characters of the Iwahori-Hecke algebras of type $A$ that extends the result of Ram.

As in the Macdonald's book [6], symmetric functions play a crucial role in the representation theory of symmetric groups. Especially, the transition matrix $M(p, s)$ from power sum functions to Schur functions is the character table of the symmetric group. Combinatorial rules to compute character values such as Murnaghan-Nakayama formula have been given by making use of properties of symmetric functions. On the other hand, various generalizations of symmetric functions are defined until now. Hall-Littlewood functions are symmetric functions with one parameter which intermediate between monomial symmetric functions and Schur functions. Among several types of Hall-Littlewood functions, The one which is denoted by $q_{\lambda}(x, q)$ yields the Frobenius formula for the characters of the Iwahori-Hecke algebra ([10]).

In order to give a super Frobenius formula for the Iwahori-Hecke algebra, we define the Hall-Littlewood supersymmetric functions $P_{\lambda}(x / y ; q)$ and $q_{\lambda}(x / y, q)$ and investigate some properties of them in section 2 . Let $x=\left(x_{1}, x_{2}, \ldots, x_{m}\right)$ and $y=\left(y_{1}, y_{2}, \ldots, y_{n}\right)$ be commutative variables. Using a partition of unity in $\mathscr{H}_{\mathbb{Q}(q), r}(q)$, that is a complete set of orthogonal minimal idempotents which is specialized to a partition of unity in $\mathbb{C}\left[\mathfrak{S}_{r}\right]$, we derive that the trace of the product of $\pi_{r}(h) \in \mathcal{A}_{q}$ and $D_{r} \in \mathcal{B}_{q}$ is R.H.S. of the super Frobenius formula (Definitions of $\pi_{r}, \mathcal{A}_{q}, D_{r}, \mathcal{B}_{q}$ are given in section 3 ).

Theorem 4.4. For any $h \in \mathscr{H}_{\mathbb{Q}(q)(x, y), r}(q)$,

$$
\operatorname{tr}\left(D_{r} \pi_{r}(h)\right)=\sum_{\lambda \vdash r} \chi^{\lambda}(h) s_{\lambda}(x / y),
$$

where $\chi^{\lambda}$ is the irreducible character of $\mathscr{H}_{\mathbb{Q}(q)(x, y), r}(q)$ corresponding to $\lambda$.

We investigate L.H.S. of Theorem 4.4 in section 5. We obtain that when $h$ is the element $T_{\gamma_{k}}$ of $\mathscr{H}_{\mathbb{Q}(q)(x, y), r}(q)$ corresponding to the cycle permutation of length $k$, L.H.S. coincides with $q_{k}\left(x / y ; q^{-2}\right)$ up to constant.

Theorem 5.3.

$$
\operatorname{tr}\left(D_{k} \pi_{k}\left(T_{\gamma_{k}}\right)\right)=\frac{q^{k}}{q-q^{-1}} q_{k}\left(x / y ; q^{-2}\right) .
$$

Finally, for the product $T_{\gamma_{\mu}}=T_{\gamma_{\mu_{1}}} T_{\gamma_{\mu_{2}}} \cdots T_{\gamma_{\mu_{l}}}$ where $\mu=\left(\mu_{1}, \mu_{2}, \ldots\right) \vdash r$, we have

Theorem 5.5. For $\mu \vdash r$,

$$
\frac{q^{|\mu|}}{\left(q-q^{-1}\right)^{l(\mu)}} q_{\mu}\left(x / y ; q^{-2}\right)=\sum_{\lambda \vdash r} \chi^{\lambda}\left(T_{\gamma_{\mu}}\right) s_{\lambda}(x / y) .
$$

Though not all the values of characters can be computed by Theorem 5.5, owing to our version of Ram's result

Theorem 5.6 ([10], Theorem 5.1). For each $T_{\sigma}, \sigma \in \mathfrak{S}_{r}$, there exists a $\mathbb{Z}\left[q, q^{-1}\right]$ linear combination

$$
c_{\sigma}=\sum_{\mu \vdash r} a_{\sigma \mu} T_{\gamma_{\mu}}
$$


$a_{\sigma \mu} \in \mathbb{Z}\left[q, q^{-1}\right]$, such that $\chi\left(T_{\sigma}\right)=\chi\left(c_{\sigma}\right)$ for all characters $\chi$ of $\mathscr{H}_{\mathbb{Q}(q), r}(q)$,

shows that any character of $\mathscr{H}_{\mathbb{Q}(q), r}(q)$ is determined by its values on the elements $T_{\gamma_{\mu}}$.

Our result extends [10, and may yield new combinatorial rules for computing the irreducible characters of Iwahori-Hecke algebras. Moreover, as we have pointed out in [8], the super version of the representation theory of the symmetric group and the Iwahori-Hecke algebra are more suitable to describe the representation theory of the alternating group and its $q$-analogue. Our result will be used to derive a Frobenius formula for the $q$-analogue of the alternating group which was defined in 7 .

\section{Preliminaries on supersymmetric functions}

Symmetric functions, besides their own interest, play important roles in various areas in mathematics. Particularly, the relation between symmetric functions and the representation theory is intimate. In this section, we will give supersymmetrizations of various classes of symmetric functions and investigate relationships between them. The basic reference of symmetric functions and supersymmetric functions is Macdonald's book [6]. We will follow [6] with respect to our notation about symmetric functions unless otherwise stated.

We denote by $\mathfrak{S}_{m}$ the symmetric group of order $m$ !. Let $\Lambda_{m}=\mathbb{Z}\left[x_{1}, x_{2}, \ldots, x_{m}\right]^{\mathfrak{S}_{m}}$ be the ring of symmetric functions of $m$ variables and $\left(\Lambda_{m}\right)_{\mathbb{Q}}=\Lambda_{m} \otimes_{\mathbb{Z}} \mathbb{Q}$. One can define an involution

$$
\omega: \Lambda_{m} \longrightarrow \Lambda_{m}
$$

by $\omega\left(e_{r}\right)=h_{r}$. Then, as [6],I,(2.13),(3.8), $\omega\left(p_{r}\right)=(-1)^{r-1} p_{r}$ and $\omega\left(s_{\lambda}\right)=s_{\lambda^{\prime}}$ hold. Let $x=$ $\left(x_{1}, x_{2}, \ldots x_{m}\right)$ be $m$ variables and $y=\left(y_{1}, y_{2}, \ldots y_{n}\right) n$ variables. We denote by $f(x, y)$ a symmetric function $f\left(x_{1}, x_{2}, \ldots, x_{m}, y_{1}, y_{2}, \ldots, y_{n}\right) \in \Lambda_{m+n}$ for abbreviation. According to [], I.3,ex.23, we set

$$
E_{x / y}(t)=\prod_{i=1}^{m}\left(1+x_{i} t\right) / \prod_{j=1}^{n}\left(1+y_{j} t\right) .
$$

The elementary supersymmetric functions $e_{k}(x / y)(k \geq 0)$ is defined by

$$
E_{x / y}(t)=\sum_{k \geq 0} e_{k}(x / y) t^{k}
$$

From $(2.1),(2.2), e_{k}(x / y)$ is a polynomial in $x=\left(x_{1}, x_{2}, \ldots x_{m}\right)$ and $y=\left(y_{1}, y_{2}, \ldots, y_{n}\right)$, symmetric in each set of variables separately. We denote by $\Lambda_{m, n}$ the ring of polynomials in $x_{1}, x_{2}, \ldots x_{m}, y_{1}, y_{2}, \ldots y_{n}$, which are separately symmetric in $x^{\prime} s$ and $y^{\prime} s$, namely

$$
\Lambda_{m, n}=\mathbb{Z}\left[x_{1}, x_{2}, \ldots, x_{m}\right]^{\mathfrak{S}_{m}} \otimes_{\mathbb{Z}} \mathbb{Z}\left[y_{1}, y_{2}, \ldots, y_{n}\right]^{\mathfrak{S}_{n}} .
$$

We also denote by $\left(\Lambda_{m, n}\right)_{\mathbb{Q}}=\Lambda_{m, n} \otimes_{\mathbb{Z}} \mathbb{Q}$. $\omega$ can be regarded as an involution on $\Lambda_{m, n}$ which acts on each 
factor of $\mathbb{Z}\left[x_{1}, x_{2}, \ldots, x_{m}\right]^{\mathfrak{S}_{m}} \otimes_{\mathbb{Z}} \mathbb{Z}\left[y_{1}, y_{2}, \ldots, y_{n}\right]^{\mathfrak{S}_{n}}$ as $\omega$. One can deduce from (2.1),(2.2) that

$$
\begin{aligned}
e_{k}(x / y) & =\sum_{i+j=k}(-1)^{j} e_{i}(x) h_{j}(y) \quad([6], \text { I. } 3, \text { ex.23 }) \\
& =\sum_{i+j=k} e_{i}(x) h_{j}(-y)
\end{aligned}
$$

Let $\omega_{y}$ be the involution $\omega$ acting on symmetric functions of $y$ variables. Then, $e_{k}(x / y)$ is obtained from $\omega_{y} e_{k}(x, y)$ by replacing each $y_{i}$ by $-y_{i}$. For each partition $\lambda$, supersymmetric Schur function $s_{\lambda}(x / y)$ is defined by

$$
s_{\lambda}(x / y)=\operatorname{det}\left(e_{\lambda_{i}^{\prime}-i+j}(x / y)\right)_{1 \leq i, j \leq l}
$$

where $l=l\left(\lambda^{\prime}\right)$. We readily see that

$$
e_{k}(x / y)=s_{\left(1^{k}\right)}(x / y)
$$

By making use of the equations

$$
\begin{gathered}
\omega s_{\lambda / \mu}(x)=s_{\lambda^{\prime} / \mu^{\prime}}(x) \quad([\underline{6}, \mathrm{I},(5.6)), \\
s_{\lambda}(x, y)=\sum_{\mu \subset \lambda} s_{\mu}(x) s_{\lambda / \mu}(y) \quad([6], \mathrm{I},(5.9)),
\end{gathered}
$$

and $\omega_{y}$, one can obtain

$$
\begin{aligned}
s_{\lambda}(x / y) & =\sum_{\mu \subset \lambda}(-1)^{|\lambda-\mu|} s_{\mu}(x) s_{\lambda^{\prime} / \mu^{\prime}}(y) \quad([6], \mathrm{I} .5, \text { ex.23(1)) } \\
& =\sum_{\mu \subset \lambda} s_{\mu}(x) s_{\lambda^{\prime} / \mu^{\prime}}(-y) .
\end{aligned}
$$

In a similar manner to $(2.1)$, we define $H_{x / y}(t)$ to be

$$
H_{x / y}(t)=\prod_{i=1}^{m}\left(1-x_{i} t\right)^{-1} \prod_{j=1}^{n}\left(1-y_{j} t\right)
$$

and the completely supersymmetric functions $h_{k}(x / y)(k \geq 0)$ to be

$$
H_{x / y}(t)=\sum_{k \geq 0} h_{k}(x / y) t^{k}
$$

One can deduce from $(2.4),(2.5)$ that

$$
h_{k}(x / y)=\sum_{i+j=k} h_{i}(x) e_{j}(-y)=e_{k}(-y /-x) .
$$

Moreover, applying $\omega$ to $h_{k}(x / y)$, we have

$$
\omega h_{k}(x / y)=\sum_{i+j=k} \omega h_{i}(x) \omega e_{j}(-y)=\sum_{i+j=k} e_{i}(x) h_{j}(-y)=e_{k}(x / y)=s_{\left(1^{k}\right)}(x / y) .
$$


Hence we obtain

$$
h_{k}(x / y)=\omega s_{\left(1^{k}\right)}(x / y)=s_{(k)}(x / y) .
$$

We may also define the power sum supersymmetric functions $p_{k}(x / y)(k \geq 1)$ as follows.

$$
\begin{aligned}
p_{k}(x / y) & =\omega_{y} p_{k}(x,-y) \\
& =p_{k}(x)+(-1)^{k-1} p_{k}(-y) \\
& =p_{k}(x)-p_{k}(y)
\end{aligned}
$$

In the same way as $[6], \mathrm{I},(2.10)$, we obtain the following relation for the generating function $P_{x / y}(t)$.

$$
\begin{aligned}
P_{x / y}(t) & =\sum_{r \geq 1} p_{r}(x / y) t^{r-1} \\
& =\sum_{r \geq 1} \sum_{i=1}^{m} x_{i}^{r} t^{r-1}-\sum_{r \geq 1} \sum_{i=1}^{n} y_{i}^{r} t^{r-1} \\
& =\sum_{i=1}^{m} \frac{x_{i}}{1-x_{i} t}-\sum_{i=1}^{n} \frac{y_{i}}{1-y_{i} t} \\
& =\sum_{i=1}^{m} \frac{d}{d t} \log \frac{1}{1-x_{i} t}-\sum_{i=1}^{n} \frac{d}{d t} \log \frac{1}{1-y_{i} t} \\
& =\frac{d}{d t} \log \prod_{i=1}^{m}\left(1-x_{i} t\right)^{-1} \prod_{j=1}^{n}\left(1-y_{j} t\right) \\
& =\frac{d}{d t} \log H_{x / y}(t)=\frac{H_{x / y}^{\prime}(t)}{H_{x / y}(t)} .
\end{aligned}
$$

Thus, we have

$$
\begin{aligned}
H_{x / y}(t) & =\exp \left(\sum_{r \geq 1} p_{r}(x / y) \frac{t^{r}}{r}\right) \\
& =\prod_{r \geq 1} \exp \left(p_{r}(x / y) \frac{t^{r}}{r}\right) \\
& =\prod_{r \geq 1} \sum_{m_{r} \geq 0} \frac{\left(p_{r}(x / y) t^{r}\right)^{m_{r}}}{m_{r} ! r^{m_{r}}},
\end{aligned}
$$

where $m_{i}$ is the number of appearances of $i$ in $\lambda_{1}, \lambda_{2}, \ldots, \lambda_{l(\lambda)}$. Let $\lambda$ ? $=\prod_{i \geq 1} i^{m_{i}} m_{i} !$. Then

$$
H_{x / y}(t)=\sum_{\lambda} \frac{p_{\lambda}(x / y)}{\lambda ?} t^{|\lambda|}
$$

summed over all partitions $\lambda$. On the other hand,

$$
H_{x / y}(t)=\sum_{k \geq 0} h_{k}(x / y) t^{k}
$$


so we can conclude that

$$
h_{k}(x / y)=\sum_{\lambda \vdash k} \frac{p_{\lambda}(x / y)}{\lambda ?} .
$$

We notice $e_{r}(x / y)$ and $h_{r}(x / y)$ are defined to be zero if $r<0$.

By an analogy of the case of symmetric functions, we will use the following equation later to give a super version of the Frobenius formula for the characters of Iwahori-Hecke algebras.

Theorem 2.1 (Super Jacobi-Trudi formula, 11, (1.9)). Let $\lambda \vdash r$. Then

$$
s_{\lambda}(x / y)=\operatorname{det}\left[h_{\lambda_{i}-i+j}(x / y)\right]_{1 \leq i, j \leq r}
$$

In 11, the above formula is given for the hook-Schur function

$$
H S_{\lambda}(x ; y)=\sum_{\mu \subset \lambda} s_{\mu}(x) s_{\lambda^{\prime} / \mu^{\prime}}(y),
$$

which is different from $s_{\lambda}(x / y)$ slightly. But replacing $y$ with $-y$, Theorem 2.1 is obtained from [11] immediately. Super Jacobi-Trudi formula is also found in [9].

Let $\Lambda_{m, n}^{r}$ be the subspace of $\Lambda_{m, n}$ consisting of $r$ homogeneous polynomials. For arbitrary $r \in \mathbb{N}$, we define a map $\psi$ from $\mathfrak{S}_{r}$ to $\Lambda_{m, n}^{r}$ as follows.

$$
\psi(w)=p_{\rho(w)}(x / y)=p_{\rho_{1}}(x / y) p_{\rho_{2}}(x / y) \cdots p_{\rho_{l(\rho)}}(x / y)
$$

where $\rho(w)=\left(\rho_{1}, \rho_{2}, \ldots \rho_{l(\rho)}\right)$ is the cycle type of $w$. Let $v \times w \in \mathfrak{S}_{r_{1}} \times \mathfrak{S}_{r_{2}}$. Then $v \times w$ can be embedded in $\mathfrak{S}_{r_{1}+r_{2}}$ in many ways. Nevertheless, for the same reason as in I.7 in [6], we have

$$
\psi(v \times w)=\psi(v) \psi(w) .
$$

In general, for functions $f, g$ on a finite group $G$ which values in a commutative $\mathbb{Q}$-algebra, the scalar product of $f$ and $g$ is defined by

$$
\langle f, g\rangle=\frac{1}{|G|} \sum_{x \in G} f(x) g\left(x^{-1}\right) .
$$

Let $\operatorname{Irr}(G)$ be a basic set of irreducible $\mathbb{C}$-characters of $G$ and $\operatorname{ch}(\mathbb{C}[G])=\oplus_{\zeta \in \operatorname{Irr}} \mathbb{Z} \zeta$ the $\mathbb{Z}$-module of virtual characters. For $f_{i} \in \operatorname{Irr}\left(\mathfrak{S}_{r_{i}}\right)(i=1,2)$, we define $f_{1} \cdot f_{2} \in \operatorname{ch}\left(\mathbb{C}\left[\mathfrak{S}_{r_{1}+r_{2}}\right]\right)$ to be

$$
f_{1} \cdot f_{2}=\operatorname{ind}_{\mathfrak{S}_{r_{1}} \times \mathfrak{S}_{r_{2}}}^{\mathfrak{S}_{r_{1}+r_{2}}}\left(f_{1} \times f_{2}\right)
$$

Then, as in [6],I.7, the $\mathbb{Z}$-module

$$
\operatorname{ch}(\mathbb{C}[\mathfrak{S}])=\bigoplus_{r \geq 0} \operatorname{ch}\left(\mathbb{C}\left[\mathfrak{S}_{r}\right]\right)
$$

has a ring structure with the product (2.7). By an analogy of the characteristic map as in [6],I.7, we define a $\mathbb{Z}$-linear map

$$
\operatorname{sch}: \operatorname{ch}(\mathbb{C}[\mathfrak{S}]) \longrightarrow\left(\Lambda_{m, n}\right)_{\mathbb{C}}=\Lambda_{m, n} \otimes \mathbb{C}
$$


to be

$$
\operatorname{sch}(f)=\langle f, \psi\rangle_{\mathfrak{S}_{r}}=\frac{1}{r !} \sum_{w \in \mathfrak{S}_{r}} f(w) \psi(w)=\sum_{\mu \vdash r} f(\mu) \frac{p_{\mu}(x / y)}{\mu ?},
$$

where $f(\mu)$ is the value of $f$ at elements of cycle type $\mu$. Using Frobenius reciprocity, we obtain

$$
\begin{aligned}
\operatorname{sch}\left(f_{1} \cdot f_{2}\right) & =\left\langle\operatorname{ind}_{\mathfrak{S}_{r_{1}} \times \mathfrak{S}_{r_{2}}}^{\mathfrak{S}_{r_{1}+r_{2}}}\left(f_{1} \times f_{2}\right), \psi\right\rangle_{\mathfrak{S}_{r_{1}+r_{2}}} \\
& =\left\langle\left(f_{1} \times f_{2}\right), \operatorname{res}_{\mathfrak{S}_{r_{1}} \times \mathfrak{S}_{r_{2}}}^{\mathfrak{S}_{r+r_{2}}}(\psi)\right\rangle_{\mathfrak{S}_{r_{1}} \times \mathfrak{S}_{r_{2}}} \\
& =\frac{1}{\left|\mathfrak{S}_{r_{1}}\right|\left|\mathfrak{S}_{r_{2}}\right|} \sum_{v \times w \in \mathfrak{S}_{r_{1}} \times \mathfrak{S}_{r_{2}}}\left(f_{1} \times f_{2}\right)(v \times w) \psi(v \times w) \\
& =\frac{1}{\left|\mathfrak{S}_{r_{1}}\right|\left|\mathfrak{S}_{r_{2}}\right|} \sum_{v \in \mathfrak{S}_{r_{1}}, w \in \mathfrak{S}_{r_{2}}} f_{1}(v) f_{2}(w) \psi(v) \psi(w) \\
& =\left\langle f_{1}, \psi\right\rangle_{\mathfrak{S}_{r_{1}}}\left\langle f_{2}, \psi\right\rangle_{\mathfrak{S}_{r_{2}}} \\
& =\operatorname{sch}\left(f_{1}\right) \operatorname{sch}\left(f_{2}\right) .
\end{aligned}
$$

Therefore sch is a ring homomorphism.

\section{Proposition 2.2.}

$$
s_{\lambda}(x / y)=\sum_{\mu \vdash r} \chi^{\lambda}(\mu) \frac{p_{\mu}(x / y)}{\mu ?},
$$

where $\chi^{\lambda}$ is the irreducible character of $\mathbb{C}\left[\mathfrak{S}_{r}\right]$ corresponding to $\lambda$.

Proof. Let $\eta_{r}$ be the identity character of $\mathfrak{S}_{r}$ and $\eta_{r}=0$ if $r<0$. Then by (2.6),

$$
\operatorname{sch}\left(\eta_{r}\right)=\sum_{\mu \vdash r} \frac{p_{\mu}(x / y)}{\mu ?}=h_{r}(x / y) .
$$

For $\lambda=\left(\lambda_{1}, \lambda_{2}, \ldots, \lambda_{l}\right) \vdash r(l=l(\lambda))$, let $\eta_{\lambda}=\eta_{\lambda_{1}} \cdot \eta_{\lambda_{2}} \cdots \cdots \eta_{\lambda_{l}}$ be the character

$$
\eta_{\lambda}=\operatorname{ind}_{\mathfrak{S}_{\lambda_{1}} \times \mathfrak{S}_{\lambda_{1}} \times \cdots \times \mathfrak{S}_{\lambda_{l}}}^{\mathfrak{S}_{r}}\left(\eta_{\lambda_{1}} \times \eta_{\lambda_{2}} \times \cdots \times \eta_{\lambda_{\lambda_{l}}}\right)
$$

of $\mathfrak{S}_{r}$. Then we have $\operatorname{sch}\left(\eta_{\lambda}\right)=h_{\lambda}(x / y)$. From [6],I,(7.4),(7.6)(i), $\chi^{\lambda}=\operatorname{det}\left[\eta_{\lambda_{i}-i+j}\right]_{1 \leq i, j \leq r}$. Using Theorem 2.1 one can deduce

$$
\operatorname{sch}\left(\chi^{\lambda}\right)=\operatorname{det}\left[h_{\lambda_{i}-i+j}(x / y)\right]_{1 \leq i, j \leq r}=s_{\lambda}(x / y),
$$

while the definition of sch yields

$$
\operatorname{sch}\left(\chi^{\lambda}\right)=\sum_{\mu \vdash r} \chi^{\lambda}(\mu) \frac{p_{\mu}(x / y)}{\mu ?} .
$$


Let

$$
v_{m}(q)=\prod_{i=1}^{m} \frac{1-q^{i}}{1-q}
$$

and

$$
v_{\lambda}(q)=\prod_{i=1}^{l} v_{\lambda_{i}}(q)
$$

for $\lambda=\left(\lambda_{1}, \lambda_{2}, \ldots \lambda_{m}\right) \vdash r, l(\lambda) \leq m$, (in which some of the $\lambda_{i}$ may be zero). The Hall-Littlewood symmetric function $P_{\lambda}(x ; q)$ for the variables $x=\left(x_{1}, x_{2}, \ldots, x_{m}\right)$ is defined by $([\underline{6}, \mathrm{III},(2.1))$

$$
P_{\lambda}(x ; q)=\frac{1}{v_{\lambda}(q)} \sum_{w \in \mathfrak{S}_{m}} w\left(x_{1}^{\lambda_{1}} x_{2}^{\lambda_{2}} \cdots x_{m}^{\lambda_{m}} \prod_{i<j} \frac{x_{i}-q x_{j}}{x_{i}-x_{j}}\right) .
$$

$P_{\lambda}(x ; q)$ is defined to be zero if $l(\lambda)>m . P_{\lambda}(x ; q), l(\lambda) \leq m$ constitute a basis of $\left(\Lambda_{m}\right)_{\mathbb{Q}(q)}=\Lambda_{m} \otimes_{\mathbb{Z}} \mathbb{Q}(q)$. Let $\varphi_{r}(q)=\prod_{i=1}^{r}\left(1-q^{i}\right)$ and

$$
b_{\lambda}(q)=\prod_{i \geq 1} \varphi_{m_{i}(\lambda)}(q),
$$

where $m_{i}(\lambda)=\#\left\{j \mid \lambda_{j}=i\right\}$. Another Hall-Littlewood symmetric function $Q_{\lambda}(x ; q)$ is defined by $([\underline{6}, \mathrm{III},(2.11))$

$$
Q_{\lambda}(x ; q)=b_{\lambda}(q) P_{\lambda}(x ; q) .
$$

$P_{\lambda}(x ; q)$ and $Q_{\lambda}(x ; q)$ are homogeneous of degree $|\lambda|$. Furthermore, two other symmetric functions $q_{r}(x ; q)$ and $q_{\lambda}(x ; q)$ for a partition $\lambda=\left(\lambda_{1}, \lambda_{2}, \ldots, \lambda_{l(\lambda)}\right)$ are defined by

$$
\begin{aligned}
& q_{0}(x ; q)=1=P_{0}(x ; q) \\
& q_{r}(x ; q)=(1-q) P_{(r)}(x ; q)=(1-q) \sum_{i=1}^{m} x_{i}^{r} \prod_{j \neq i} \frac{x_{i}-q x_{j}}{x_{i}-x_{j}} \quad(r \geq 1), \\
& q_{\lambda}(x ; q)=\prod_{i=1}^{l(\lambda)} q_{\lambda_{i}}(x ; q) .
\end{aligned}
$$

As in [6],III, $(4.8)$, we may define a scalar product on $\left(\Lambda_{m}\right)_{\mathbb{Q}(q)}$ by requiring that the bases $\left(q_{\lambda}(x ; q)\right)$ and $\left(m_{\lambda}(x)\right)$ be dual to each other:

$$
\left\langle q_{\lambda}(x ; q), m_{\mu}(x)\right\rangle=\delta_{\lambda, \mu}
$$

Then one can see

$$
\left\langle P_{\lambda}(x ; q), Q_{\lambda}(x ; q)\right\rangle=\delta_{\lambda, \mu} \quad([\underline{6}, \mathrm{III},(4.9)) .
$$

The generating function $Q(u)$ of $q_{r}(x ; q)$ is

$$
Q(u)=\sum_{r \geq 0} q_{r}(x ; q) u^{r}=\prod_{i=1}^{m} \frac{1-x_{i} q u}{1-x_{i} u} \quad([\underline{6}, \mathrm{III},(2.10)) .
$$


In the similar manner as (2.1), we define a supersymmetric function $q_{r}(x / y ; q) \in \Lambda_{m, n}$ as follows.

$$
Q_{x / y}(u)=\sum_{r \geq 0} q_{r}(x / y ; q) u^{r}=\prod_{i=1}^{m} \frac{1-x_{i} q u}{1-x_{i} u} \prod_{j=1}^{n} \frac{1-y_{j} u}{1-y_{j} q u} .
$$

From the definition of $q_{r}(x / y ; q)$, we immediately have

$$
q_{r}(x / y ; q)=\sum_{k=0}^{r} q_{k}(x ; q) q_{r-k}\left(q y ; q^{-1}\right)=\sum_{k=0}^{r} q^{r-k} q_{k}(x ; q) q_{r-k}\left(y ; q^{-1}\right) .
$$

In order to define the Hall-Littlewood supersymmetric functions, we shall give a definition of the skew Hall-Littlewood symmetric functions $P_{\lambda / \mu}(x ; q)$ according to [6],III,5. $P_{\lambda / \mu}(x ; q)$ is a symmetric function which is uniquely determined by

$$
\left\langle P_{\lambda / \mu}(x ; q), Q_{\nu}(x ; q)\right\rangle=\left\langle P_{\lambda}(x ; q), Q_{\mu}(x ; q) Q_{\nu}(x ; q)\right\rangle
$$

More constructive way of definition is as follows. For two partitions $\lambda, \mu$ such that $\lambda-\mu=\theta$ is a horizontal strip, let $J_{\theta}=\left\{j \in \mathbb{N} \mid \theta_{j}^{\prime}<\theta_{j+1}^{\prime}\right\}$ and

$$
\psi_{\lambda / \mu}(q)=\prod_{j \in J_{\theta}}\left(1-q^{m_{j}(\mu)}\right)
$$

where $m_{j}(\mu)=\#\left\{i \mid \mu_{i}=j\right\}$. For general $\lambda, \mu$, take a semi-standard tableau (it is called "column-strict tableau" or simply "tableau" in [6] $) T=\left(\mu=\lambda_{0} \subset \lambda_{1} \subset \cdots \subset \lambda_{l}=\lambda\right)$ and set

$$
\psi_{T}(q)=\prod_{i=1}^{l} \psi_{\lambda_{i} / \lambda_{i-1}}(q) .
$$

Then $P_{\lambda / \mu}(x ; q)$ is defined by

$$
P_{\lambda / \mu}(x ; q)=\sum_{T} \psi_{T}(q) x^{T}
$$

summed over all semi-standard tableau $T$ of shape $\lambda-\mu$.

Lemma 2.3. For $0<k \leq r$,

$$
P_{(r) /(k)}(x ; q)=\sum_{\nu \vdash r-k}(1-q)^{l(\nu)} m_{\nu}(x) .
$$

Proof. Let $\lambda=(r)$ and $\mu=(k)$, and consider a semi-standard tableau $T=\left(\mu=\lambda_{0} \subset \lambda_{1} \subset \cdots \subset \lambda_{l}=\lambda\right)$. In each $\psi_{\lambda_{i} / \lambda_{i-1}}(q)$, we readily see that $J_{\theta}=\left\{\left|\lambda_{i-1}\right|\right\}$ and $m_{\left|\lambda_{i-1}\right|}\left(\lambda_{i-1}\right)=1$ if $\lambda_{i-1} \subsetneq \lambda_{i}$ and $J_{\theta}=\phi$ if $\lambda_{i-1}=\lambda_{i}$. Therefore, for each semi-standard tableau $T, \psi_{T}(q)=(1-q)^{\#\left\{i \mid \lambda_{i-1} \subsetneq \lambda_{i}\right\}}$. Summing up all semi-standard tableaux, we obtain the equation.

Lemma 2.4.

$$
\begin{aligned}
& P_{(r) / 0}(x ; q)=P_{(r)}(x ; q), \\
& P_{(r) /(r)}(x ; q)=1 \\
& P_{(r) /(k)}(x ; q)=(1-q) P_{(r-k)}(x ; q) \quad(0<k<r) .
\end{aligned}
$$


Proof. The first equation is direct consequence of (2.9). The second is obtained from Lemma 2.3. For the third, we use [6],III.2,Ex.3,

$$
P_{(r)}(x ; q)=\sum_{i=0}^{r-1}(-q)^{i} s_{\left(r-i, 1^{i}\right)}(x) .
$$

Since the transition matrix $M(s, m)$ equals to $\left(K_{\lambda \mu}\right)$ consisting of Kostka numbers, the numbers of semi-standard tableaux of shape $\lambda$ and weight $\mu$, we have

$$
P_{(r-k)}(x ; q)=\sum_{i=0}^{r-k-1}(-q)^{i} \sum_{\nu \vdash r-k} K_{\left(r-k-i, 1^{i}\right) \nu} m_{\nu}(x) .
$$

By [6],I.6,Ex.2(b),

$$
K_{\left(r-k-i, 1^{i}\right) \nu}=\left(\begin{array}{c}
l(\nu)-1 \\
i
\end{array}\right) .
$$

So, observing that $l(\nu)-1 \leq r-k-1$, and that if $l(\nu)-1<i$ then $\underset{i}{l(\nu)-1})=0$, we obtain

$$
P_{(r-k)}(x ; q)=\sum_{\nu \vdash r-k} \sum_{i=0}^{l(\nu)-1}(-q)^{i}\left(\begin{array}{c}
l(\nu)-1 \\
i
\end{array}\right) m_{\nu}(x) .
$$

On the other hand, from Lemma 2.3,

$$
\begin{aligned}
P_{(r) /(k)}(x ; q) & =\sum_{\nu \vdash r-k}(1-q)^{l(\nu)} m_{\nu}(x) \\
& =(1-q) \sum_{\nu \vdash r-k}(1-q)^{l(\nu)-1} m_{\nu}(x) \\
& =(1-q) \sum_{\nu \vdash r-k}\left\{\sum_{i=0}^{l(\nu)-1}\left(\begin{array}{c}
l(\nu)-1 \\
i
\end{array}\right)(-q)^{i}\right\} m_{\nu}(x) .
\end{aligned}
$$

Lemma 2.5. For $r \geq 1$,

$$
\omega P_{(r)}(x ; q)=(-1)^{r-1} q^{-1} P_{(r)}\left(q x ; q^{-1}\right)
$$


Proof. From (2.25), we have

$$
\begin{aligned}
\omega P_{(r)}(x ; q) & =\omega \sum_{i=0}^{r-1}(-q)^{i} s_{\left(r-i, 1^{i}\right)}(x) \\
& =\sum_{i=0}^{r-1}(-q)^{i} s_{\left(r-i, 1^{i}\right)^{\prime}}(x) \\
& =\sum_{i=0}^{r-1}(-q)^{i} s_{\left(i+1,1^{r-i-1}\right)}(x) \\
& =\sum_{j=0}^{r-1}(-q)^{r-1-j} s_{\left(r-j, 1^{j}\right)}(x) \\
& =(-q)^{r-1} \sum_{j=0}^{r-1}(-q)^{-j} s_{\left(r-j, 1^{j}\right)}(x) \\
& =(-q)^{r-1} P_{(r)}\left(x ; q^{-1}\right) \\
& =(-1)^{r-1} q^{-1} P_{(r)}\left(q x ; q^{-1}\right)
\end{aligned}
$$

It is known $\left([6], \mathrm{III},\left(5.5^{\prime}\right)\right)$ that

$$
P_{\lambda}(x, y ; q)=\sum_{\mu \subset \lambda} P_{\mu}(x ; q) P_{\lambda / \mu}(y ; q) .
$$

We define the Hall-Littlewood supersymmetric functions $P_{\lambda}(x / y ; q)$ to be

$$
P_{\lambda}(x / y ; q)=\omega_{y} P_{\lambda}(x,-y ; q)=\sum_{\mu \subset \lambda} P_{\mu}(x ; q) \omega P_{\lambda / \mu}(-y ; q) .
$$

When $q=0$, R.H.S. of (2.10) reduces to that of (2.3). Hence we obtain $P_{\lambda}(x / y ; 0)=s_{\lambda}(x / y)$.

\section{Proposition 2.6.}

$$
\begin{aligned}
& P_{0}(x / y ; q)=q_{0}(x / y ; q) \\
& P_{(r)}(x / y ; q)=\frac{1}{1-q} q_{r}(x / y ; q) \quad(r>0) .
\end{aligned}
$$


Proof. The first equation is obvious. From Lemma 2.4, Lemma 2.5 and (2.10), for $r>0$ we have

$$
\begin{aligned}
P_{(r)}(x / y ; q) & =\sum_{k=0}^{r} P_{(k)}(x ; q) \omega P_{(r) /(k)}(-y ; q) \\
& =\omega P_{(r) /(0)}(-y ; q)+\sum_{k=1}^{r-1} P_{(k)}(x ; q) \omega P_{(r) /(k)}(-y ; q)+P_{(r)}(x ; q) \\
& =(-1)^{r} \omega P_{(r)}(y ; q)+\sum_{k=1}^{r-1} P_{(k)}(x ; q)(-1)^{r-k}(1-q) \omega P_{(r-k)}(y ; q)+P_{(r)}(x ; q) \\
& =(-q)^{-1} P_{(r)}\left(q y ; q^{-1}\right)+\sum_{k=1}^{r-1} P_{(k)}(x ; q)\left(1-q^{-1}\right) P_{(r-k)}\left(q y ; q^{-1}\right)+P_{(r)}(x ; q) \\
& =\frac{1}{1-q} q_{r}\left(q y ; q^{-1}\right)+\sum_{k=1}^{r-1} \frac{1}{1-q} q_{k}(x ; q) q_{r-k}\left(q y ; q^{-1}\right)+\frac{1}{1-q} q_{r}(x ; q) \\
& =\frac{1}{1-q} q_{r}(x / y ; q) .
\end{aligned}
$$

\section{Iwahori-Hecke algebras and general quantum super algebras}

In this section, we review the sign $q$-permutation representation of the Iwahori-Hecke algebra $\mathscr{H}_{q}$ of type $A\left([\underline{8})\right.$, and the vector representation of the general quantum super algebra $U_{q}^{\sigma}([\underline{1},[])$.

Let $R$ be a commutative domain with 1 , and let $q$ be an invertible element of $R$. The Iwahori-Hecke algebra $\mathscr{H}_{R, r}(q)$ of type $A$ is an $R$-algebra generated by $\left\{T_{i} \mid i=1,2, \ldots, r-1\right\}$ with the relations:

(H1) $T_{i}^{2}=\left(q-q^{-1}\right) T_{i}+1 \quad$ if $i=1,2, \ldots, r-1$,

(H2) $T_{i} T_{i+1} T_{i}=T_{i+1} T_{i} T_{i+1} \quad$ if $i=1,2, \ldots, r-2$,

(H3) $T_{i} T_{j}=T_{j} T_{i} \quad$ if $|i-j|>1$.

Let $R=\mathbb{Z}\left[q, q^{-1}\right]$ be a polynomial ring over $\mathbb{Z}$ with indeterminates $q^{ \pm 1}$, and $K$ the quotient field of $R$. Let $M=\oplus_{k=1}^{m+n} R v_{k}$ be a $\mathbb{Z}_{2}$-graded $R$-module of rank $m+n$. By $\mathbb{Z}_{2}$-graded, we mean that $M$ is a direct sum of two submodule $M_{\overline{0}}=\oplus_{k=1}^{m} R v_{k}$ and $M_{\overline{1}}=\oplus_{k=m+1}^{m+n} R v_{k}$, and that for each homogeneous element the degree map $|\cdot|$

$$
|v|= \begin{cases}0 & \text { if } v \in M_{\overline{0}} \\ 1 & \text { if } v \in M_{\overline{1}}\end{cases}
$$

is given. 
Let $\pi_{r}$ be the $q$-permutation representation of $\mathscr{H}_{R, r}(q)$ on the tensor space $M^{\otimes r}$. As defined in [8], $\pi_{r}$ is given by $\pi_{r}\left(T_{i}\right)=\mathrm{Id}^{\otimes i-1} \otimes T \otimes \mathrm{Id}^{\otimes r-i-1}(i=1,2, \ldots, r-1)$ where $T$ is the operator on $M \otimes M$ defined by

$$
T v_{k} \otimes v_{l}= \begin{cases}\frac{(-1)^{\left|v_{k}\right|}\left(q+q^{-1}\right)+q-q^{-1}}{2} v_{k} \otimes v_{l} & \text { if } k=l, \\ (-1)^{\left|v_{k}\right|\left|v_{l}\right|} v_{l} \otimes v_{k}+\left(q-q^{-1}\right) v_{k} \otimes v_{l} & \text { if } k<l, \\ (-1)^{\left|v_{k}\right|\left|v_{l}\right|} v_{l} \otimes v_{k} & \text { if } k>l .\end{cases}
$$

and Id is the identity operator on $M$. This representation $\pi_{r}$ is reduced to the (normal) $q$-permutation representation of $\mathscr{H}_{R, r}(q)$ with $n=0$ and to the sign permutation representation of the symmetric group $\mathfrak{S}_{r}$ with $q \rightarrow 1$. For abbreviation, we denote $\mathscr{H}_{q}=\mathscr{H}_{K, r}(q)=K \otimes_{R} \mathscr{H}_{R, r}(q)$ and set $V=K \otimes_{R} M$. The above action also defines a representation of $\mathscr{H}_{q}$ on $V^{\otimes r}$.

Next, we define the quantum superalgebra $U_{q}^{\sigma}(\mathfrak{g r}(m, n))$ and its vector representation on the tensor space $V^{\otimes r}$ according to [1. $U_{q}^{\sigma}(\mathfrak{g l}(m, n))$ is a Hopf algebra obtained from the quantum superalgebra $U_{q}(\mathfrak{g l}(m, n))$, which is a Hopf superalgebra, by adding an involutive element $\sigma$.

Let $P=\oplus_{b \in B} \mathbb{Z} \epsilon_{b}$ be a free $\mathbb{Z}$-module where $B=B_{+} \cup B_{-}$with $B_{+}=\{1, \ldots, m\}$ and $B_{-}=\{m+$ $1, \ldots, m+n\}$, and $\Pi=\left\{\alpha_{i}=\epsilon_{i}-\epsilon_{i+1}\right\}_{i \in I}$ a set of simple roots with the index set $I=I_{\text {even }} \cup I_{\text {odd }}$ where $I_{\text {even }}=\{1,2, \ldots, m-1, m+1, \ldots, m+n-1\}$ and $I_{\text {odd }}=\{m\}$. We define a map $p: I \longrightarrow\{0,1\}$ to be such that

$$
p(i)= \begin{cases}0 & \text { if } i \in I_{\text {even }}, \\ 1 & \text { if } i \in I_{\text {odd }}\end{cases}
$$

A $\mathbb{Q}$-valued symmetric bilinear form on $P(\cdot, \cdot): P \times P \longrightarrow \mathbb{Q}$ is defined as follows.

$$
\left(\epsilon_{a}, \epsilon_{a^{\prime}}\right)= \begin{cases}1 & \text { if } a=a^{\prime} \in B_{+} \\ -1 & \text { if } a=a^{\prime} \in B_{-} \\ 0 & \text { otherwise }\end{cases}
$$

The set $\Pi^{\vee}=\left\{h_{i} \mid i \in I\right\}$ of simple coroots is uniquely determined by the formula $\ell_{i}\left\langle h_{i}, \lambda\right\rangle=\left(\alpha_{i}, \lambda\right)$ for any $\lambda \in P$, where $\langle\cdot, \cdot\rangle$ is the natural pairing $\langle\cdot, \cdot\rangle: P^{*} \times P \longrightarrow \mathbb{Z}$ between $P$ and $P^{*}$ and

$$
\ell_{i}= \begin{cases}1 & \text { if } i=1, \ldots, m, \\ -1 & \text { if } i=m+1, \ldots, m+n-1\end{cases}
$$

The quantized enveloping algebra $U_{q}^{\sigma}(\mathfrak{g l}(m, n))$ is the unital associative algebra over $K$ with generators $q^{h}\left(h \in P^{*}\right), e_{i}, f_{i}(i \in I)$ and an additional element $\sigma$ which satisfy the following defining relations:

(Q1) $q^{h}=1 \quad$ for $h=0$,

(Q2) $q^{h_{1}} q^{h_{2}}=q^{h_{1}+h_{2}} \quad$ for $h_{1}, h_{2} \in P^{*}$,

(Q3) $q^{h} e_{i}=q^{\left\langle h, \alpha_{j}\right\rangle} e_{i} q^{h} \quad$ for $h \in P^{*}$ and $i \in I$, 
(Q4) $q^{h} f_{i}=q^{-\left\langle h, \alpha_{j}\right\rangle} f_{i} q^{h} \quad$ for $h \in P^{*}$ and $i \in I$,

(Q5) $\left[e_{i}, f_{j}\right]=\delta_{i j} \frac{q^{\ell_{i} h_{i}}-q^{-\ell_{i} h_{i}}}{q^{\ell_{i}}-q^{-\ell_{i}}} \quad$ for $i, j \in I$,

(Q6) $\sigma^{2}=1$,

(Q7) $q^{h} \sigma=\sigma q^{h} \quad$ for $h \in P^{*}$,

(Q8) $e_{i} \sigma=(-1)^{p(i)} \sigma e_{i} \quad$ for $i \in I$,

(Q9) $f_{i} \sigma=(-1)^{p(i)} \sigma f_{i} \quad$ for $i \in I$,

where $\left[e_{i}, f_{j}\right]$ means the supercommutator

$$
\left[e_{i}, f_{j}\right]=e_{i} f_{j}-(-1)^{p(i) p(j)} f_{j} e_{i} .
$$

We assume further conditions (bitransitivity condition, see [5] p.19):

(Q10) If $a \in \sum_{i \in I} U_{q}\left(\mathfrak{n}_{+}\right) e_{i} U_{q}\left(\mathfrak{n}_{+}\right)$satisfies $f_{i} a \in U_{q}\left(\mathfrak{n}_{+}\right) f_{i}$ for all $i \in I$, then $a=0$,

(Q11) If $a \in \sum_{i \in I} U_{q}\left(\mathfrak{n}_{-}\right) f_{i} U_{q}\left(\mathfrak{n}_{-}\right)$satisfies $e_{i} a \in U_{q}\left(\mathfrak{n}_{-}\right) e_{i}$ for all $i \in I$, then $a=0$,

where $U_{q}\left(\mathfrak{n}_{+}\right)$(respectively $\left.U_{q}\left(\mathfrak{n}_{-}\right)\right)$is the subalgebra of $U_{q}^{\sigma}(\mathfrak{g l}(m, n))$ generated by $\left\{e_{i} \mid i \in I\right\}$ (respectively $\left.\left\{f_{i} \mid i \in I\right\}\right) . U_{q}^{\sigma}(\mathfrak{g l}(m, n))$ is a Hopf algebra whose comultiplication $\triangle$, counit $\varepsilon$, antipode $S$ are as follows.

$$
\begin{aligned}
& \triangle(\sigma)=\sigma \otimes \sigma, \\
& \triangle\left(q^{h}\right)=q^{h} \otimes q^{h} \quad \text { for } h \in P^{*}, \\
& \triangle\left(e_{i}\right)=e_{i} \otimes q^{-\ell_{i} h_{i}}+\sigma^{p(i)} \otimes e_{i} \quad \text { for } i \in I, \\
& \triangle\left(f_{i}\right)=f_{i} \otimes 1+\sigma^{p(i)} q^{\ell_{i} h_{i}} \otimes f_{i} \quad \text { for } i \in I, \\
& \varepsilon(\sigma)=\varepsilon\left(q^{h}\right)=1 \quad \text { for } h \in P^{*}, \quad \varepsilon\left(e_{i}\right)=\varepsilon\left(f_{i}\right)=0 \quad \text { for } i \in I, \\
& S(\sigma)=\sigma, \quad S\left(q^{ \pm h}\right)=q^{\mp h} \quad \text { for } h \in P^{*}, \\
& S\left(e_{i}\right)=-\sigma^{p(i)} e_{i} q^{\ell_{i} h_{i}}, \quad S\left(f_{i}\right)=-\sigma^{p(i)} q^{-\ell_{i} h_{i}} f_{i} \quad \text { for } i \in I .
\end{aligned}
$$

For the sake of abbreviation, we denote $U_{q}^{\sigma}(\mathfrak{g l}(m, n))$ by $U_{q}^{\sigma}$. The vector representation $(\rho, V)$ of $U_{q}^{\sigma}$ on $\mathbb{Z}_{2}$-graded vector space $V=V_{\overline{0}} \oplus V_{\overline{1}}$ (recall that $V_{\overline{0}}=\oplus_{i=1}^{m} R v_{i}, V_{\overline{1}}=\oplus_{i=m+1}^{m+n} R v_{i}$ ) is defined by

$$
\begin{aligned}
& \rho(\sigma) v_{j}=(-1)^{\left|v_{j}\right|} v_{j} \quad \text { for } j=1, \ldots, m+n, \\
& \rho\left(q^{h}\right) v_{j}=q^{\left\langle h, \epsilon_{j}\right\rangle} v_{j} \quad \text { for } h \in P^{*}, j=1, \ldots, m+n, \\
& \rho\left(e_{j}\right) v_{j+1}=v_{j} \quad \text { for } j=1, \ldots, m+n-1, \\
& \rho\left(f_{j}\right) v_{j}=v_{j+1} \quad \text { for } j=1, \ldots, m+n-1,
\end{aligned}
$$

otherwise 0 . 
This representation can be extended to the representation on the tensor space $V^{\otimes r}$. Let $\rho_{r}$ be the map from $U_{q}^{\sigma}$ to $\operatorname{End}_{K}\left(V^{\otimes r}\right)$ defined by

$$
\begin{aligned}
& \rho_{r}(\sigma)=\rho(\sigma)^{\otimes r}, \\
& \rho_{r}\left(q^{h}\right)=\rho\left(q^{h}\right)^{\otimes r} \quad \text { for } h \in P^{*}, \\
& \rho_{r}\left(e_{i}\right)=\sum_{k=1}^{N} \rho\left(\sigma^{p(i)}\right)^{\otimes k-1} \otimes \rho\left(e_{i}\right) \otimes \rho\left(q^{-\ell_{i} h_{i}}\right)^{\otimes r-k} \quad \text { for } i \in I, \\
& \rho_{r}\left(f_{i}\right)=\sum_{k=1}^{r} \rho\left(\sigma^{p(i)} q^{\ell_{i} h_{i}}\right)^{\otimes k-1} \otimes \rho\left(f_{i}\right) \otimes \mathrm{Id}^{\otimes r-k} \quad \text { for } i \in I .
\end{aligned}
$$

Let $\triangle^{(1)}=\triangle$ and set $\triangle^{(k)}=\left(\triangle \otimes \mathrm{Id}^{\otimes k-1}\right) \triangle^{(k-1)}$ inductively. Then from the definition of $\triangle$, we have $\rho_{r}(g)=\rho^{\otimes r} \circ \triangle^{(r-1)}(g)$ for $g \in U_{q}^{\sigma}$ immediately.

Proposition 3.1 (1] Proposition 3.1). $\rho_{r}$ gives a completely reducible representation of $U_{q}^{\sigma}$ on $V^{\otimes r}$ for $r \geq 1$.

We denote $\pi_{r}\left(\mathscr{H}_{q}\right)$ by $\mathcal{A}_{q}$ and $\rho_{r}\left(U_{q}^{\sigma}\right)$ by $\mathcal{B}_{q}$. In $[8]$, we have shown that $\mathcal{A}_{q}$ and $\mathcal{B}_{q}$ are full centralizers of each other in $\operatorname{End}_{K} V^{\otimes r}$. Namely,

Theorem 3.2 ([8] Theorem 4.4). $\operatorname{End}_{\mathcal{B}_{q}} V^{\otimes r}=\mathcal{A}_{q}$ and $\operatorname{End}_{\mathcal{A}_{q}} V^{\otimes r}=\mathcal{B}_{q}$.

We denote by $\bar{K}$ the algebraic closure of $K$. We set $\overline{\mathscr{H}}_{q}=\mathscr{H}_{q} \otimes_{K} \bar{K}, \bar{U}_{q}^{\sigma}=U_{q}^{\sigma} \otimes_{K} \bar{K}, \overline{\mathcal{A}}_{q}=\mathcal{A}_{q} \otimes_{K} \bar{K}$, $\overline{\mathcal{B}}_{q}=\mathcal{B}_{q} \otimes_{K} \bar{K}$. Then, $\pi_{r}\left(\overline{\mathscr{H}}_{q}\right)=\overline{\mathcal{A}}_{q}$ and $\rho_{r}\left(\bar{U}_{q}^{\sigma}\right)=\overline{\mathcal{B}}_{q}$ as $\bar{K}$-algebras of operators on $\bar{V}^{\otimes r}=\left(V \otimes_{K} \bar{K}\right)^{\otimes r}$. Let $H(m, n ; r)=\left\{\lambda=\left(\lambda_{1}, \lambda_{2}, \ldots\right) \vdash r \mid \lambda_{j} \leq n\right.$ if $\left.j>m\right\}$. Diagrams of elements of $H(m, n ; r)$ are exactly those contained by the $(m, n)$-hooks.

Theorem 3.3 ([8] Theorem 5.1). $\bar{V}^{\otimes r}=\bigoplus_{\lambda \in H(m, n ; r)} H_{\lambda} \otimes V_{\lambda}$ where, $H_{\lambda}(\lambda \in H(m, n ; r))$ are mutually non-isomorphic simple $\overline{\mathscr{H}}_{q}$-modules, $V_{\lambda}(\lambda \in H(m, n ; r))$ are mutually non-isomorphic simple $\bar{U}_{q}^{\sigma}$-modules.

In fact, Theorem 3.3 holds over $K$ since it is known that $K$ is a splitting field for $\mathscr{H}_{q}$. Hence $\mathscr{H}_{q}$ is a split semisimple $K$-algebra, and we do not need to take the algebraic closure.

\section{Traces of the actions of $\mathscr{H}_{q}$ and $U_{q}^{\sigma}$}

In this section, we introduce indeterminates $x_{1}, \ldots, x_{m}, y_{1}, \ldots, y_{n}$ associated to the basis $v_{1}, \ldots, v_{m+n}$ and consider the trace of the product of two operators $D_{r} \in \mathcal{B}_{q}$ and $\pi_{r}(h) \in \mathcal{A}_{q}$ with $h \in \mathscr{H}_{q}$, in the same manner as in [10] or [13. Using a partition of unity of $\mathscr{H}_{q}$ which has been given in [3] and [14] independently, we obtain that the trace generates characters of $\mathscr{H}_{q}$. Regarding $\mathbb{C}\left[\mathfrak{S}_{r}\right]$ as the specialization of $\mathscr{H}_{q}$ to 1 , we use same notations such as $\pi_{r}, \chi^{\lambda}$,etc., for $\mathbb{C}\left[\mathfrak{S}_{r}\right]$. 
Let $K^{\prime}=K\left(z_{1}, z_{2}, \ldots, z_{m+n}\right)$ be the field of rational functions on $K$. In the remainder of this paper, we assume that $\mathscr{H}_{q}, U_{q}^{\sigma}, V$, etc., are defined over $K^{\prime}$ and use the same notations such as $\mathscr{H}_{q}, U_{q}^{\sigma}$, $V$, etc.. We notice that Proposition 3.1, Theorem 3,2, Theorem 3.3 are hold for $K^{\prime}$. Let

$$
\begin{aligned}
& \mathscr{I}_{r, m+n}=\left\{\mathbf{i}=\left(i_{1}, i_{2}, \ldots, i_{r}\right) \mid 1 \leq i_{k} \leq m+n\right\} \\
& \mathscr{I}_{r, m+n}^{+}=\left\{\mathbf{i}=\left(i_{1}, i_{2}, \ldots, i_{r}\right) \mid 1 \leq i_{k} \leq m+n, i_{1} \leq i_{2} \leq \cdots \leq i_{r}\right\}
\end{aligned}
$$

and

$$
\mathscr{C}_{r, m+n}=\left\{\mathbf{c}=\left(c_{1}, c_{2}, \ldots, c_{m+n}\right) \mid c_{k} \geq 0, \sum c_{k}=r\right\} .
$$

For $\mathbf{i}=\left(i_{1}, i_{2}, \ldots, i_{r}\right) \in \mathscr{I}_{r, m+n}$, we define $c(\mathbf{i})=\left(c_{1}, c_{2}, \ldots, c_{m+n}\right)$ where $k$ appears $c_{k}$ times in $i_{1}, i_{2}, \ldots, i_{r}$ for each $k=1,2, \ldots, m+n$. Clearly $c$ maps $\mathscr{I}_{r, m+n}$ onto $\mathscr{C}_{r, m+n}$. Let $E_{i} \in \operatorname{End}_{K^{\prime}} V(i=1,2, \ldots m+n)$ be the projections,

$$
E_{i} v_{k}=\delta_{i k} v_{k}
$$

We set $v_{\mathbf{i}}=v_{i_{1}} \otimes v_{i_{2}} \otimes \cdots \otimes v_{i_{r}}$ and

$$
E_{\mathbf{c}}=\sum_{c(\mathbf{i})=\mathbf{c}} E_{i_{1}} \otimes \cdots \otimes E_{i_{r}}
$$

for $\mathbf{c} \in \mathscr{C}_{r, m+n}$. Then $E_{\mathbf{c}}$ is the projection from $V^{\otimes r}$ to the subspace $V_{\mathbf{c}}^{\otimes r}$ which is defined by

$$
V_{\mathbf{c}}^{\otimes r}=\sum_{c(\mathbf{i})=\mathbf{c}} K^{\prime} v_{\mathbf{i}}
$$

By an easy calculation, we see that $E_{i} \otimes E_{j}+E_{j} \otimes E_{i}(i, j=1,2, \ldots, m+n)$ commute with $T$ on $V^{\otimes 2}$. For each term

$$
E_{i_{1}} \otimes \cdots \otimes E_{i_{k-1}} \otimes E_{i_{k}} \otimes E_{i_{k+1}} \otimes E_{i_{k+2}} \otimes \cdots \otimes E_{i_{r}}
$$

of $E_{\mathbf{c}}$, there exists a term such that

$$
E_{i_{1}} \otimes \cdots \otimes E_{i_{k-1}} \otimes E_{i_{k+1}} \otimes E_{i_{k}} \otimes E_{i_{k+2}} \otimes \cdots \otimes E_{i_{r}}
$$

in $E_{\mathbf{c}}$. Hence $E_{\mathbf{c}}$ commutes with the action of $\mathscr{H}_{q}$, namely, $E_{\mathbf{c}}$ belongs to $\operatorname{End}_{\mathcal{A}_{q}} V^{\otimes r}=\mathcal{B}_{q}=\rho_{r}\left(U_{q}^{\sigma}\right)$. Let $z^{\mathbf{c}}=z_{1}^{c_{1}} z_{2}^{c_{2}} \cdots z_{m+n}^{c_{m+n}}$ and define an operator $D_{r}$ on $V^{\otimes r}$ by

$$
D_{r}=\sum_{\mathbf{c} \in \mathscr{C}_{r, m+n}} z^{\mathbf{c}} E_{\mathbf{c}}
$$

As mentioned above, we have $D_{r} \in \operatorname{End}_{\mathcal{A}_{q}} V^{\otimes r}$. In the same manner as in the proof of Lemma 3.5 in [10], one can show:

Lemma 4.1 ([10] Lemma 3.5). For any idempotent $p \in \mathscr{H}_{q}, \operatorname{tr}\left(D_{r} \pi_{r}(p)\right)$ is independent of $q$.

Let us define the specialization to a nonzero complex number $t$ to be a ring homomorphism $\varphi_{t}: R \longrightarrow \mathbb{C}$ with the condition $\varphi_{t}(q)=t$. $\mathbb{C}$ becomes $(\mathbb{C}, R)$-bimodule, with $R$ acting from the right via $\varphi_{t}$. By 
the specialization $\varphi_{t}$, one has $\mathbb{C} \otimes_{R} \mathscr{H}_{R, r}(q) \cong \mathscr{H}_{\mathbb{C}, r}(t)$, especially, if $t=1$ then $\mathbb{C} \otimes_{R} \mathscr{H}_{R, r}(q) \cong \mathbb{C}\left[\mathfrak{S}_{r}\right]$. We denote by $M_{t}=\mathbb{C} \otimes_{R} M$ the specialization of $M$ by $\varphi_{t}$. If $t$ is a transcendental number, then $K \cong \mathbb{Q}(t)$ as fields via $\mathbb{Q}$-homomorphism $q \mapsto t$. Therefore, we have $\bar{K} \cong \overline{\mathbb{Q}(t)} \cong \mathbb{C}$ as fields. Let $\mathbb{C}^{\prime}=$ $\mathbb{C}\left(z_{1}, z_{2}, \ldots, z_{m+n}\right)$. Then $\mathscr{H}_{\mathbb{C}^{\prime}, r}(t)$ is a split semisimple $\mathbb{C}^{\prime}$-algebra. We may assume that $M_{1}$ is defined over $\mathbb{C}^{\prime}$. We replace $z_{1}, z_{2}, \ldots, z_{m}$ by $x_{1}, x_{2}, \ldots, x_{m}$ and $z_{m+1}, z_{m+2}, \ldots, z_{m+n}$ by $-y_{1},-y_{2}, \ldots,-y_{n}$. Then we have the following two lemmas in a similar way as in the proofs of Lemma 3.6 and Lemma 3.7 in [10].

Lemma 4.2. Let $\gamma_{r}=s_{1} s_{2} \cdots s_{r-2} s_{r-1} \in \mathfrak{S}_{r}$. Then

$$
\operatorname{tr}\left(D_{r} \pi_{r}\left(\gamma_{r}\right)\right)=\sum_{i=1}^{m} x_{i}^{r}+(-1)^{r-1} \sum_{i=1}^{n}\left(-y_{i}\right)^{r}=p_{r}(x)-p_{r}(y)=p_{r}(x / y)
$$

where $D_{r} \pi_{r}\left(\gamma_{r}\right)$ is regarded as an operator on $M_{1}^{\otimes r}$.

Proof. Since

$$
D_{r} \pi_{r}\left(\gamma_{r}\right) v_{i_{1}} \otimes v_{i_{2}} \otimes \cdots \otimes v_{i_{r}}=D_{r}(-1)^{\left|v_{i_{r}}\right|\left(\left|v_{i_{1}}\right|+\left|v_{i_{2}}\right|+\cdots+\left|v_{i_{r-1}}\right|\right)} v_{i_{r}} \otimes v_{i_{1}} \otimes \cdots \otimes v_{i_{r-1}}
$$

the coefficient of $v_{i_{1}} \otimes v_{i_{2}} \otimes \cdots \otimes v_{i_{r}}$ in $D_{r} \pi_{r}\left(\gamma_{r}\right) v_{i_{1}} \otimes v_{i_{2}} \otimes \cdots \otimes v_{i_{r}}$ is as follows.

$$
\text { coef. }=\left\{\begin{array}{l}
x_{i_{1}}^{r} \quad \text { if } i_{1}=i_{2}=\cdots=i_{r} \leq m \\
(-1)^{r-1}\left(-y_{i_{1}}\right)^{r} \quad \text { if } i_{1}=i_{2}=\cdots=i_{r}>m \\
0 \quad \text { otherwise. }
\end{array}\right.
$$

Lemma 4.3. Let $p_{\lambda}$ be a minimal idempotent of $\mathbb{C}\left[\mathfrak{S}_{r}\right]$ corresponding to a simple left $\mathbb{C}\left[\mathfrak{S}_{r}\right]$-module indexed by $\lambda \vdash r$. Then

$$
\operatorname{tr}\left(D_{r} \pi_{r}\left(p_{\lambda}\right)\right)=s_{\lambda}(x / y)
$$

where $s_{\lambda}(x / y)$ is the supersymmetric Schur function.

Proof. Let $z_{\lambda}$ be the minimal central idempotent of $\mathbb{C}\left[\mathfrak{S}_{r}\right]$ indexed by $\lambda$, and $\chi^{\lambda}$ the character which is afforded by $\mathbb{C}\left[\mathfrak{S}_{r}\right] p_{\lambda} . z_{\lambda}$ is given by

$$
z_{\lambda}=\frac{d_{\lambda}}{r !} \sum_{\sigma \in \mathfrak{S}_{r}} \chi^{\lambda}(\sigma) \sigma .
$$

Let $d_{\lambda}$ be the degree of $\mathbb{C}\left[\mathfrak{S}_{r}\right] p_{\lambda} . \mathbb{C}\left[\mathfrak{S}_{r}\right]$ contains just $d_{\lambda}$ simple left $\mathbb{C}\left[\mathfrak{S}_{r}\right]$-modules which are isomorphic to $\mathbb{C}\left[\mathfrak{S}_{r}\right] p_{\lambda}$. Thus we have the following.

$$
\begin{aligned}
\operatorname{tr}\left(D_{r} \pi_{r}\left(p_{\lambda}\right)\right) & =\frac{1}{d_{\lambda}} \operatorname{tr}\left(D_{r} \pi_{r}\left(z_{\lambda}\right)\right) \\
& =\frac{1}{d_{\lambda}} \frac{d_{\lambda}}{r !} \sum_{\sigma \in \mathfrak{S}_{r}} \chi^{\lambda}(\sigma) \operatorname{tr}\left(D_{r} \pi_{r}(\sigma)\right) .
\end{aligned}
$$


Let $\mu=\left(\mu_{1}, \mu_{2}, \ldots \mu_{l}\right) \vdash r$ where $l=l(\mu)$ is the length of $\mu$. We set cycle permutations as follows.

$$
\begin{aligned}
\gamma_{\mu_{1}} & =\left(12 \ldots \mu_{1}\right) \\
\gamma_{\mu_{2}} & =\left(\mu_{1}+1 \mu_{1}+2 \ldots \mu_{1}+\mu_{2}\right) \\
& \vdots \\
\gamma_{\mu_{l}} & =\left(\mu_{1}+\cdots+\mu_{l-1}+1 \mu_{1}+\cdots+\mu_{l-1}+2 \ldots r\right) \\
\gamma_{\mu} & =\gamma_{\mu_{1}} \gamma_{\mu_{2}} \cdots \gamma_{\mu_{l}}
\end{aligned}
$$

Since $\chi^{\lambda}(\sigma)$ and $\operatorname{tr}\left(D_{r} \pi_{r}(\sigma)\right)$ depend only upon the cycle type $\mu$ of $\sigma$, they are constant on conjugacy classes $C_{\mu}$. Hence,

$$
\begin{aligned}
\operatorname{tr}\left(D_{r} \pi_{r}\left(p_{\lambda}\right)\right) & =\frac{1}{r !} \sum_{\mu \vdash r} \chi^{\lambda}(\mu) \operatorname{tr}\left(D_{r} \pi_{r}\left(\gamma_{\mu}\right)\right)\left|C_{\mu}\right| \\
& =\frac{1}{r !} \sum_{\mu \vdash r} \chi^{\lambda}(\mu) \prod_{k=1}^{l(\mu)} \operatorname{tr}\left(D_{r} \pi_{r}\left(\gamma_{\mu_{k}}\right)\right)\left|C_{\mu}\right| \\
& =\frac{1}{r !} \sum_{\mu \vdash r} \chi^{\lambda}(\mu) \prod_{k=1}^{l(\mu)} p_{\mu_{k}}(x / y)\left|C_{\mu}\right| \\
& =\sum_{\mu \vdash r} \chi^{\lambda}(\mu) \frac{p_{\mu}(x / y)}{\mu ?} .
\end{aligned}
$$

From Proposition 2.2, we have $\operatorname{tr}\left(D_{r} \pi_{r}\left(p_{\lambda}\right)\right)=s_{\lambda}(x / y)$ as desired.

In the same way as in the proof of Theorem 3.8 in [10, we have

Theorem 4.4. For any $h \in \mathscr{H}_{q}$,

$$
\operatorname{tr}\left(D_{r} \pi_{r}(h)\right)=\sum_{\lambda \vdash r} \chi^{\lambda}(h) s_{\lambda}(x / y),
$$

where $\chi^{\lambda}$ is the irreducible character of $\mathscr{H}_{q}$ corresponding to $\lambda$.

Proof. Let $\left\{p_{i}^{\lambda} \mid \lambda \vdash r, i=1,2, \ldots, d_{\lambda}=\operatorname{dim} V_{\lambda}\right\}$ be a partition of unity in $\mathscr{H}_{q}$ such that when we apply the specialization $\varphi_{1}, p_{i}^{\lambda}$ are well-defined and yield a partition of unity in $\mathbb{C}\left[\mathfrak{S}_{r}\right]$. Then we have

$$
\begin{aligned}
\operatorname{tr}\left(D_{r} \pi_{r}(h)\right) & =\sum_{\lambda, \mu \vdash r} \sum_{i=1}^{d_{\lambda}} \sum_{j=1}^{d_{\mu}} \operatorname{tr}\left(D_{r} \pi_{r}\left(p_{i}^{\lambda} h p_{j}^{\mu}\right)\right) \\
& =\sum_{\lambda, \mu \vdash r} \sum_{i=1}^{d_{\lambda}} \operatorname{tr}\left(D_{r} \pi_{r}\left(p_{i}^{\lambda} h p_{i}^{\lambda}\right)\right) \\
& =\sum_{\lambda \vdash r} \sum_{i=1}^{d_{\lambda}} h_{i i}^{\lambda} \operatorname{tr}\left(D_{r} \pi_{r}\left(p_{i}^{\lambda}\right)\right),
\end{aligned}
$$


where $h_{i i}^{\lambda}$ is the diagonal element of the representation matrix of $h$ in the irreducible representation corresponding to $\lambda$ determined by this partition of unity. Thus by Lemma 4.3, we obtain

$$
\begin{aligned}
\operatorname{tr}\left(D_{r} \pi_{r}(h)\right) & =\sum_{\lambda \vdash r} \sum_{i=1}^{d_{\lambda}} h_{i i}^{\lambda} s_{\lambda}(x / y) \\
& =\sum_{\lambda \vdash r} \chi^{\lambda}(h) s_{\lambda}(x / y)
\end{aligned}
$$

\section{The super Frobenius formula for the characters of $\mathscr{H}_{q}$}

In this section, we compute $\operatorname{tr}\left(D_{r} \pi_{r}(h)\right)$ in detail. We shall show that when $h$ is a product of elements corresponding to cycle permutations, the trace coincides with a Hall-Littlewood supersymmetric function up to constant. There is no notion of cycle type for elements of $\mathscr{H}_{q}$ in general, so not all character values are given in this way. Nevertheless, by Ram's result, any character of $\mathscr{H}_{q}$ is determined by its values on elements corresponding to cycle permutations.

For $\mathbf{i}=\left(i_{1}, i_{2}, \ldots i_{r}\right) \in \mathscr{I}_{r, m+n}$, we define cardinalities of subsets of $\left\{i_{1}, i_{2}, \ldots i_{r}\right\}$ as follows.

$$
\begin{array}{rlll}
N^{0}(\mathbf{i}) & =\#\left\{j \mid i_{j} \leq m\right\}, \quad N^{1}(\mathbf{i})=\#\left\{j \mid i_{j}>m\right\}, & & \\
E^{0}(\mathbf{i})=\#\left\{j \mid i_{j}=i_{j+1}, i_{j} \leq m\right\}, & E^{1}(\mathbf{i})=\#\left\{j \mid i_{j}=i_{j+1}, i_{j}>m\right\}, & E(\mathbf{i})=E^{0}(\mathbf{i})+E^{1}(\mathbf{i}), \\
L^{0}(\mathbf{i})=\#\left\{j \mid i_{j}<i_{j+1}, i_{j} \leq m\right\}, & L^{1}(\mathbf{i})=\#\left\{j \mid i_{j}<i_{j+1}, i_{j}>m\right\}, & L(\mathbf{i})=L^{0}(\mathbf{i})+L^{1}(\mathbf{i}) .
\end{array}
$$

Let $T_{\gamma_{r}}=T_{s_{1}} T_{s_{2}} \cdots T_{s_{r-1}}$. Using above notations we obtain:

Proposition 5.1. For $1 \leq k \leq r$, the trace of $D_{k} \pi_{k}\left(T_{\gamma_{k}}\right)$ on $V^{\otimes k}$ is given by

$$
\operatorname{tr}\left(D_{k} \pi_{k}\left(T_{\gamma_{k}}\right)\right)=\sum_{\mathbf{i} \in \mathscr{I}_{k, m+n}^{+}}(-1)^{E^{1}(\mathbf{i})} q^{E^{0}(\mathbf{i})-E^{1}(\mathbf{i})}\left(q-q^{-1}\right)^{L(\mathbf{i})} z_{i_{1}} z_{i_{2}} \cdots z_{i_{k}}
$$

Proof. We prove by induction on $k$. If $k=1$, then $T_{\gamma_{1}}=1$, so the statement holds obviously. Now assume $k>1$. We consider $D_{k} \pi_{k}\left(T_{\gamma_{k}}\right) v_{i_{1}} \otimes v_{i_{2}} \otimes \cdots \otimes v_{i_{k}}$ case-by-case depending on the relation between $i_{k-1}$ and $i_{k}$.

case $1: i_{k-1}>i_{k}$

$$
D_{k} \pi_{k}\left(T_{\gamma_{k}}\right) v_{i_{1}} \otimes v_{i_{2}} \otimes \cdots \otimes v_{i_{k-1}} \otimes v_{i_{k}}=(-1)^{\left|v_{i_{k-1}}\right|\left|v_{i_{k}}\right|} D_{k} \pi_{k}\left(T_{\gamma_{k-1}}\right) v_{i_{1}} \otimes v_{i_{2}} \otimes \cdots \otimes v_{i_{k}} \otimes v_{i_{k-1}}
$$

Since $\pi_{k}\left(T_{\gamma_{k-1}}\right)$ acts on the first $k-1$ factors and $i_{k-1} \neq i_{k}$, the coefficient of $v_{i_{1}} \otimes v_{i_{2}} \otimes \cdots \otimes v_{i_{k-1}} \otimes v_{i_{k}}$ in $D_{k} \pi_{k}\left(T_{\gamma_{k}}\right) v_{i_{1}} \otimes v_{i_{2}} \otimes \cdots \otimes v_{i_{k}}$ is zero.

case $2: i_{k-1}<i_{k}$

$$
\begin{aligned}
D_{k} \pi_{k}\left(T_{\gamma_{k}}\right) v_{i_{1}} \otimes v_{i_{2}} \otimes \cdots \otimes v_{i_{k-1}} \otimes v_{i_{k}} & =(-1)^{\left|v_{i_{k-1}}\right|\left|v_{i_{k}}\right|} D_{k} \pi_{k}\left(T_{\gamma_{k-1}}\right) v_{i_{1}} \otimes v_{i_{2}} \otimes \cdots \otimes v_{i_{k}} \otimes v_{i_{k-1}} \\
& +\left(q-q^{-1}\right) D_{k} \pi_{k}\left(T_{\gamma_{k-1}}\right) v_{i_{1}} \otimes v_{i_{2}} \otimes \cdots \otimes v_{i_{k-1}} \otimes v_{i_{k}}
\end{aligned}
$$


For the same reason as case 1, the coefficient of $v_{i_{1}} \otimes v_{i_{2}} \otimes \cdots \otimes v_{i_{k-1}} \otimes v_{i_{k}}$ in the first term is zero. While the one in the second term equals to the coefficient of $v_{i_{1}} \otimes v_{i_{2}} \otimes \cdots \otimes v_{i_{k-1}}$ in $\left(q-q^{-1}\right) z_{i_{k}} D_{k-1} \pi_{k-1}\left(T_{\gamma_{k-1}}\right)$ $v_{i_{1}} \otimes v_{i_{2}} \otimes \cdots \otimes v_{i_{k-1}}$.

case $3: i_{k-1}=i_{k}$

$$
D_{k} \pi_{k}\left(T_{\gamma_{k}}\right) v_{i_{1}} \otimes v_{i_{2}} \otimes \cdots \otimes v_{i_{k-1}} \otimes v_{i_{k}}=\frac{(-1)^{\left|v_{i_{k}}\right|}\left(q+q^{-1}\right)+\left(q-q^{-1}\right)}{2} D_{k} \pi_{k}\left(T_{\gamma_{k-1}}\right) v_{i_{1}} \otimes v_{i_{2}} \otimes \cdots \otimes v_{i_{k-1}} \otimes v_{i_{k}}
$$

Thus the coefficient of $v_{i_{1}} \otimes v_{i_{2}} \otimes \cdots \otimes v_{i_{k}}$ in $D_{k} \pi_{k}\left(T_{\gamma_{k}}\right) v_{i_{1}} \otimes v_{i_{2}} \otimes \cdots \otimes v_{i_{k}}$ equals to that of $v_{i_{1}} \otimes v_{i_{2}} \otimes \cdots \otimes v_{i_{k-1}}$ in $q z_{i_{k}} D_{k-1} \pi_{k-1}\left(T_{\gamma_{k-1}}\right) v_{i_{1}} \otimes v_{i_{2}} \otimes \cdots \otimes v_{i_{k-1}}$ if $i_{k} \leq m$ and to that of $v_{i_{1}} \otimes v_{i_{2}} \otimes \cdots \otimes v_{i_{k-1}}$ in $-q^{-1} z_{i_{k}} D_{k-1}$ $\pi_{k-1}\left(T_{\gamma_{k-1}}\right) v_{i_{1}} \otimes v_{i_{2}} \otimes \cdots \otimes v_{i_{k-1}}$ if $i_{k}>m$.

By induction, the assertion follows as desired.

Replacing $z_{1}, z_{2}, \ldots, z_{m}$ by $x_{1}, x_{2}, \ldots, x_{m}$ and $z_{m+1}, z_{m+2}, \ldots, z_{m+n}$ by $-y_{1},-y_{2}, \ldots,-y_{n}$, we obtain

$$
\begin{aligned}
\operatorname{tr}\left(D_{k} \pi_{k}\left(T_{\gamma_{k}}\right)\right)= & \sum_{\mathbf{i} \in \mathscr{I}_{k, m+n}^{+}}(-1)^{E^{1}(\mathbf{i})} q^{E^{0}(\mathbf{i})-E^{1}(\mathbf{i})}\left(q-q^{-1}\right)^{L(\mathbf{i})} z_{i_{1}} z_{i_{2}} \cdots z_{i_{k}} \\
& =\sum_{\mathbf{i} \in \mathscr{I}_{k, m+n}^{+}} q^{E^{0}(\mathbf{i})}\left(q-q^{-1}\right)^{L^{0}(\mathbf{i})} z_{i_{1}} \cdots z_{i_{N^{0}(\mathbf{i})}}\left(-q^{-1}\right)^{E^{1}(\mathbf{i})}\left(q-q^{-1}\right)^{L^{1}(\mathbf{i})} z_{i_{N^{0}(\mathbf{i})+1}} \cdots z_{i_{k}} \\
& =\sum_{\mu \vdash k}\left(-q^{-1}\right)^{|\mu|-l(\mu)}\left(q-q^{-1}\right)^{l(\mu)-1} m_{\mu}(-y) \\
& +\sum_{j=1}^{k-1}\left\{\sum_{\lambda \vdash j} q^{|\lambda|-l(\lambda)}\left(q-q^{-1}\right)^{l(\lambda)} m_{\lambda}(x) \sum_{\mu \vdash k-j}\left(-q^{-1}\right)^{|\mu|-l(\mu)}\left(q-q^{-1}\right)^{l(\mu)-1} m_{\mu}(-y)\right\} \\
& +\sum_{\lambda \vdash k} q^{|\lambda|-l(\lambda)}\left(q-q^{-1}\right)^{l(\lambda)-1} m_{\lambda}(x)
\end{aligned}
$$

Let

$$
\tilde{q}_{k}(x ; q)=q^{k} \sum_{\lambda \vdash k}\left(\frac{q-q^{-1}}{q}\right)^{l(\lambda)} m_{\lambda}(x) \quad \text { if } k>0,
$$

and $\tilde{q}_{0}(x ; q)=1$. Since $m_{\lambda}\left(x_{1}, \ldots, x_{m}\right)=0$ if $l(\lambda)>m, \tilde{q}_{k}\left(x_{1}, \ldots, x_{m} ; q\right)=0$ if $m=0$ and $k>0$. From definition of $\tilde{q}_{k}(x ; q)$, one can describe the trace as follows.

$$
\operatorname{tr}\left(D_{k} \pi_{k}\left(T_{\gamma_{k}}\right)\right)=\frac{1}{q-q^{-1}} \sum_{j=0}^{k} \tilde{q}_{j}(x ; q) \tilde{q}_{k-j}\left(-y ;-q^{-1}\right) .
$$

Considering the generating function of $\tilde{q}_{r}(x ; q)$, we obtain the relation between $\tilde{q}_{r}(x ; q)$ and $q_{r}(x ; q)$ as follows.

\section{Lemma 5.2.}

$$
\tilde{q}_{r}(x ; q)=q_{r}\left(q x ; q^{-2}\right)=q^{r} q_{r}\left(x ; q^{-2}\right)
$$

This equations are found in [10, Theorem4.13 and [13],(6.11.3) in slightly different forms, but essentially the same. 
Proof. Consider the generating function of $\tilde{q}_{r}(x ; q)$.

$$
\begin{aligned}
\sum_{r \geq 0} \tilde{q}_{r}(x ; q) u^{r} & =\sum_{r \geq 0} q^{r} u^{r}\left\{\sum_{\mu \vdash r}\left(\frac{q-q^{-1}}{q}\right)^{l(\mu)} m_{\mu}(x)\right\} \\
& =\prod_{i=1}^{m}\left(\frac{q-q^{-1}}{q} \cdot \frac{1}{1-q u x_{i}}-\frac{q-q^{-1}}{q}+1\right) \\
& =\prod_{i=1}^{m} \frac{1-q^{-1} u x_{i}}{1-q u x_{i}} \\
& =\prod_{i=1}^{m} \frac{1-q^{-2} q x_{i} u}{1-q x_{i} u} \\
& =\sum_{r \geq 0} q_{r}\left(q x ; q^{-2}\right) u^{r} \\
& =\sum_{r \geq 0} q^{r} q_{r}\left(x ; q^{-2}\right) u^{r}
\end{aligned}
$$

Thus the equalities hold.

\section{Theorem 5.3.}

$$
\operatorname{tr}\left(D_{k} \pi_{k}\left(T_{\gamma_{k}}\right)\right)=\frac{q^{k}}{q-q^{-1}} q_{k}\left(x / y ; q^{-2}\right)
$$

Proof. From (5.1) and Lemma 5.2, we obtain

$$
\begin{aligned}
\operatorname{tr}\left(D_{k} \pi_{k}\left(T_{\gamma_{k}}\right)\right) & =\frac{1}{q-q^{-1}} \sum_{j=0}^{k} \tilde{q}_{j}(x ; q) \tilde{q}_{k-j}\left(-y ;-q^{-1}\right) \\
& =\frac{1}{q-q^{-1}} \sum_{j=0}^{k} q_{j}\left(q x ; q^{-2}\right) q_{k-j}\left(q^{-1} y ; q^{2}\right) .
\end{aligned}
$$

On the other hand, by (2.8), we have

$$
\begin{aligned}
q_{k}\left(x / y ; q^{-2}\right) & =\sum_{j=0}^{k} q_{j}\left(x ; q^{-2}\right) q_{k-j}\left(q^{-2} y ; q^{2}\right) \\
& =\sum_{j=0}^{k} q_{j}\left(x ; q^{-2}\right) q^{j-k} q_{k-j}\left(q^{-1} y ; q^{2}\right) \\
& =q^{-k} \sum_{j=0}^{k} q_{j}\left(q x ; q^{-2}\right) q_{k-j}\left(q^{-1} y ; q^{2}\right) .
\end{aligned}
$$

This completes the proof. 
A finite sequence $\alpha=\left(\alpha_{1}, \alpha_{2}, \ldots, \alpha_{l}\right)$ of positive integers which satisfy $\alpha_{1}+\alpha_{2}+\cdots+\alpha_{l}=r$ is said to be a composition of $r$ and denote by $\alpha=r$. We set cycle permutations for $\alpha$ as follows.

$$
\begin{aligned}
\gamma_{\alpha_{1}} & =\left(12 \ldots \alpha_{1}\right) \\
\gamma_{\alpha_{2}} & =\left(\alpha_{1}+1 \alpha_{1}+2 \ldots \alpha_{1}+\alpha_{2}\right) \\
& \vdots \\
\gamma_{\alpha_{l}} & =\left(\alpha_{1}+\cdots+\alpha_{l-1}+1 \alpha_{1}+\cdots+\alpha_{l-1}+2 \ldots r\right) \\
\gamma_{\alpha} & =\gamma_{\alpha_{1}} \gamma_{\alpha_{2}} \cdots \gamma_{\alpha_{l}}
\end{aligned}
$$

Then one can readily see that $T_{\gamma_{\alpha}}=T_{\gamma_{\alpha_{1}}} T_{\gamma_{\alpha_{2}}} \cdots T_{\gamma_{\alpha_{l}}}$.

\section{Proposition 5.4.}

$$
\operatorname{tr}\left(D_{r} \pi_{r}\left(T_{\gamma_{\alpha}}\right)\right)=\prod_{i=1}^{l} \operatorname{tr}\left(D_{\alpha_{i}} \pi_{\alpha_{i}}\left(T_{\gamma_{\alpha_{i}}}\right)\right)
$$

Proof. It is sufficient to prove for $l=2$. Let $T_{\gamma_{\alpha}}=T_{\gamma_{\alpha_{1}}} T_{\gamma_{\alpha_{2}}} . T_{\gamma_{\alpha_{1}}}$ and $T_{\gamma_{\alpha_{2}}}$ act only on $v_{i_{1}} \otimes v_{i_{2}} \otimes \cdots \otimes v_{i_{\alpha_{1}}}$ and $v_{i_{\alpha_{1}+1}} \otimes v_{i_{\alpha_{1}+2}} \otimes \cdots \otimes v_{i_{\alpha_{1}+\alpha_{2}}}$ respectively. Therefore,

$$
\begin{aligned}
D_{r} \pi_{r}\left(T_{\gamma_{\alpha_{1}}} T_{\gamma_{\alpha_{2}}}\right) v_{i_{1}} \otimes v_{i_{2}} \otimes \cdots \otimes v_{i_{r}} & =\left(D_{\alpha_{1}} \pi_{\alpha_{1}}\left(T_{\gamma_{\alpha_{1}}}\right) v_{i_{1}} \otimes v_{i_{2}} \otimes \cdots \otimes v_{i_{\alpha_{1}}}\right) \\
& \otimes\left(D_{\alpha_{2}} \pi_{\alpha_{2}}\left(T_{\gamma_{\alpha_{2}}}\right) v_{i_{\alpha_{1}+1}} \otimes v_{i_{\alpha_{1}+2}} \otimes \cdots \otimes v_{i_{\alpha_{1}+\alpha_{2}}}\right) .
\end{aligned}
$$

Thus we have proved the assertion.

Theorem 5.5. For $\mu \vdash r$,

$$
\frac{q^{|\mu|}}{\left(q-q^{-1}\right)^{l(\mu)}} q_{\mu}\left(x / y ; q^{-2}\right)=\sum_{\lambda \vdash r} \chi^{\lambda}\left(T_{\gamma_{\mu}}\right) s_{\lambda}(x / y)
$$

Proof. The assertion follows from Theorem 5.3 and Proposition 5.4.

By Theorem 5.1 in [10, with a slight change for our version, we obtain that any character of $\mathscr{H}_{q}$ is determined by its values on $T_{\gamma_{\mu}}, \mu \vdash r$.

Theorem 5.6 ([10], Theorem 5.1). For each $T_{\sigma}, \sigma \in \mathfrak{S}_{r}$, there exists a $\mathbb{Z}\left[q, q^{-1}\right]$ linear combination

$$
c_{\sigma}=\sum_{\mu \vdash r} a_{\sigma \mu} T_{\gamma_{\mu}}
$$

$a_{\sigma \mu} \in \mathbb{Z}\left[q, q^{-1}\right]$, such that $\chi\left(T_{\sigma}\right)=\chi\left(c_{\sigma}\right)$ for all characters $\chi$ of $\mathscr{H}_{q}$. 


\section{References}

[1] G. Benkart, S. Kang and M. Kashiwara, Crystal bases for the quantum superalgebra $U_{q}(\mathfrak{g l}(m, n))$, J. Amer. Math. Soc. 13(2) (2000), 295-331.

[2] A. Berele and A. Regev, Hook Young diagrams with applications to combinatorics and to representations of Lie superalgebras, Adv. Math. 64 (1987), 118-175.

[3] A. Gyoja, A q-analogue of Young symmetrizer, Osaka J. Math. 23 (1986), 841-852.

[4] M. Jimbo, A $q$-analogue of $U\left(\mathfrak{g l}_{n+1}\right)$, Hecke algebra and the Yang-Baxter equation, Lett. in Math. Phys. 11 (1986), 247-252.

[5] V. G. Kac, Lie superalgebras, Adv. in Math. 26 (1977), 8-96.

[6] I. G. Macdonald, "Symmetric Functions and Hall Polynomials, 2nd edition," Oxford Science Publications, 1995.

[7] H. Mitsuhashi, The $q$-analogue of the alternating group and its representations, J. Algebra $\mathbf{2 4 0}$ (2001), 535-558.

[8] H. Mitsuhashi, Schur-Weyl reciprocity between the quantum superalgebra and the Iwahori-Hecke algebra, Algebr. Represent. Theor. 9 (2006), 309-322.

[9] P. Pragacz and A. Thorup, On a Jacobi-Trudi identity for supersymmetric polynomials, Adv. Math. 95 (1992), 8-17.

[10] A. Ram, A Frobenius formula for the characters of the Hecke algebra, Invent. math. 106 (1991), $461-488$.

[11] J. B. Remmel, The combinatorics of $(k, l)$-hook Schur functions, Contemp. Math. 34 (1984), 253-287.

[12] A. N. Sergeev, The tensor algebra of the identity representation as a module over the Lie superalgebras $\mathfrak{G l}(n, m)$ and $Q(n)$, Math. USSR Sbornik. 51 (1985), No.2, 419-427.

[13] T. Shoji, A Frobenius formula for the characters of Ariki-Koike algebras, J. Algebra 226 (2000), $818-856$.

[14] H. Wenzl, Heck algebras of type $A_{n}$ and subfactors, Invent. Math. 92 (1988), 349-383. 